\title{
Acute Pancreatitis Following Endoscopic Ampullary Biopsies without Attempted Cannulation of the Ampulla of Vater
}

\author{
Spyridon Michopoulos ${ }^{1}$, Dimitra Kozompoli ${ }^{1}$, Sparti Ntai ${ }^{2}$, Georgios Kalantzis ${ }^{1}$, Evanthia Zampeli ${ }^{1}$ and Kalliopi Petraki ${ }^{3}$ \\ Departments of ${ }^{1}$ Gastroenterology and ${ }^{2}$ Radiology, Alexandra Hospital, Athens University Medical School, Athens, ${ }^{3}$ Department of Pathology, \\ Metropolitan Hospital, Neo Faliro, Greece
}

A 51-year-old man underwent diagnostic work-up for an abnormal-appearing ampulla of Vater. Three hours after biopsy of the ampulla, the patient presented with intense symptoms suggesting acute pancreatitis, which was later confirmed with laboratory and radiographic examinations. Other causes were excluded and the acute pancreatitis was considered a procedural complication. This is a rarely reported complication that must be taken into consideration when biopsies are performed in the ampulla of Vater. Clin Endosc 2016;49:575-578

Key Words: Acute pancreatitis; Ampullary biopsies; Ampulla of Vater

\section{INTRODUCTION}

Biopsies from the ampulla of Vater are usually essential to diagnose premalignant and malignant lesions in asymptomatic or symptomatic patients. Acute pancreatitis is an extremely unusual complication following non-thermal biopsy of the ampulla without attempted cannulation. We present this case because of its rarity and the accompanying intense clinical manifestations without further severe consequences.

\section{CASE REPORT}

A 51-year-old man underwent two gastroscopies over the prior 2 years with the indication of intermittent dyspepsia. On the last endoscopy, the ampulla of Vater was described

Received: February 26, 2016 Revised: May 7, 2016

Accepted: June 7, 2016

Correspondence: Spyridon Michopoulos

Department of Gastroenterology, Alexandra Hospital, Athens University Medical School, 6 Agias Kyriakis St Kifissia, Athens, Greece

Tel: +30-2103381387, Fax: +30-2132162895, E-mail: michosp5@gmail.com

(cc) This is an Open Access article distributed under the terms of the Creative Commons Attribution Non-Commercial License (http://creativecommons.org/ licenses/by-nc/3.0) which permits unrestricted non-commercial use, distribution, and reproduction in any medium, provided the original work is properly cited. as bulging; biopsies were performed and histological examinations revealed mild chronic, nonspecific, inflammatory mucosal lesions of the ampulla and duodenum, with regional architectural disturbances, epithelial hyperplasia, accompanying mild nuclear stratification, and atypia, not fulfilling the diagnostic criteria for epithelial dysplasia.

The patient was referred to our clinic with persistent, intermittent epigastric discomfort and pain, and we therefore scheduled a repeat examination in order to exclude an ampullary tumor. The patient was on acenocoumarol since an episode of pulmonary embolism 2 years prior. The extensive work-up after this episode did not reveal any aggravating factors for the embolism. In view of the results of the endoscopy, we advised the patient to discontinue acenocoumarol and changed to low molecular weight heparin (enoxaparin $40 \mathrm{mg}$ / day).

The patient was heterozygous for sickle cell disease (hemoglobin electrophoresis: HbS, 29.5\%; HbF, 0.6\%; HbA2, 3.6\%). The hemoglobin level before the procedure was $14.2 \mathrm{~g} / \mathrm{dL}$. He reported infrequent alcohol intake and smoking cessation 4 years prior. A side-viewing scope (Olympus TJF-145 Video Duodenoscope; Olympus, Tokyo, Japan) was used with the patient under conscious sedation (midazolam $2 \mathrm{mg}$, propofol $60 \mathrm{mg}$ ). The ampulla of Vater was examined and showed mild granularity (Fig. 1). Eight biopsies were obtained from the area 


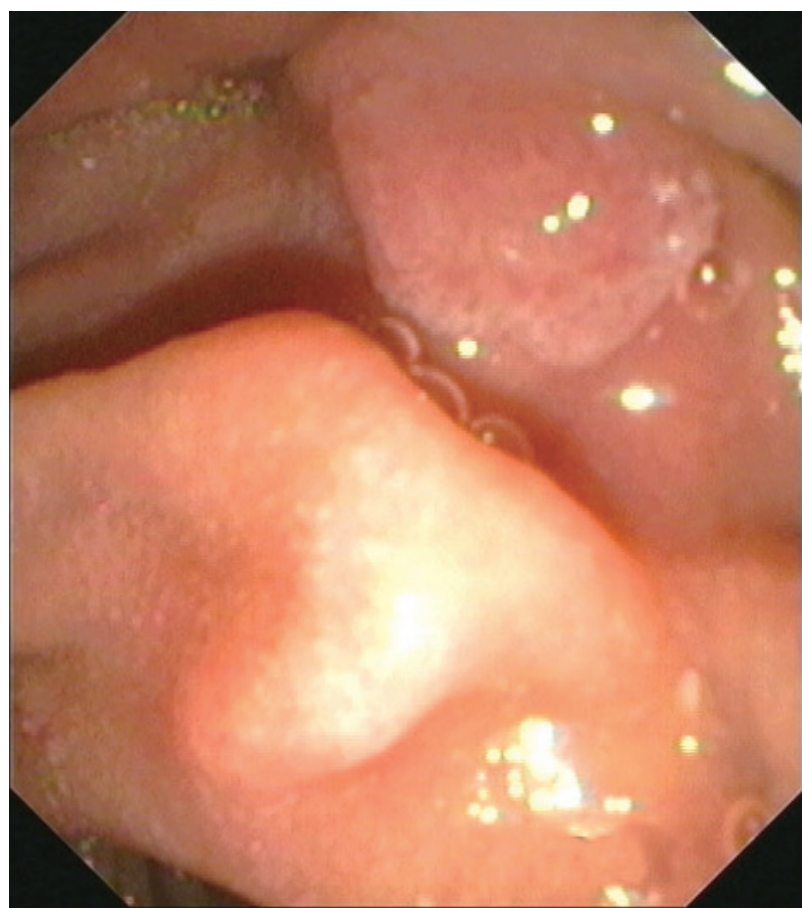

Fig. 1. Ampulla of Vater appearing mildly granular.

around the ampulla with a reusable oval-cup forceps (Olympus FB-26N-1; Olympus). No excessive bleeding was observed during endoscopy. No arrhythmia was recorded during the procedure. Recovery was uneventful and the patient went home accompanied by another person. He had a light meal 2 hours after the procedure (cheese pie).

One hour after the meal, the patient experienced an acute, severe (eight out of 10), persistent abdominal pain with recurrent, unrelieved vomiting. He contacted his family doctor but refused hospital admission and was monitored on an outpatient basis. On the second day, the patient reported less vomiting, but the pain was still intense and he additionally became febrile to $38.5^{\circ} \mathrm{C}$. He was advised to stop solid food intake and only take regular oral hydration.

On day 3 , the symptoms improved with no vomiting, milder abdominal pain, and a temperature less than $37.8^{\circ} \mathrm{C}$. Blood and urine tests were performed on the same day. Serum and urine amylase levels were $845 \mathrm{U} / \mathrm{L}$ (normal $<100$ ) and 8,500 $\mathrm{U} / \mathrm{L}$ (normal $<460$ ), respectively. Lipase testing was not available in our unit. Kidney and liver tests showed normal results except for a slight elevation of the alanine aminotransferase level. Blood triglyceride level was $110 \mathrm{mg} / \mathrm{dL}$ (normal <150) and the calcium level was $9.2 \mathrm{mg} / \mathrm{dL}$ (normal 8.5 to 10.2).

Abdominal ultrasound did not reveal cholelithiasis or other pathology. On day 5 , the symptoms completely resolved. A second abdominal ultrasound was unremarkable, while contrast-enhanced abdominal computed tomography (CT) re-
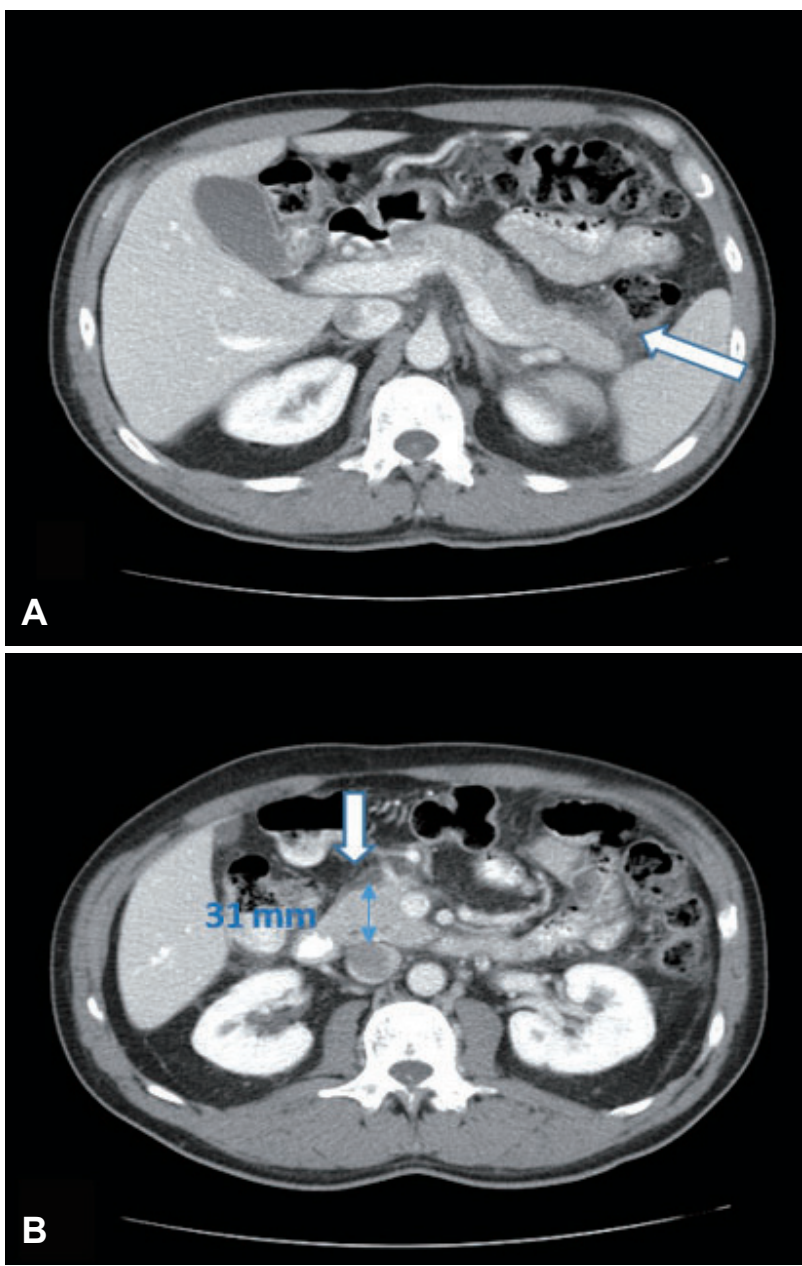

Fig. 2. (A, B) Portal phase of contrast-enhanced computed tomography of upper abdomen: stranding of retroperitoneal peripancreatic fat (arrows) and mild enlargement of pancreatic head ( $B$, anteroposterior diameter $31 \mathrm{~mm}$ ).

vealed mild enlargement of the pancreatic head and stranding of the surrounding retroperitoneal fat (Fig. 2). Since then, the patient has returned to his usual activities.

Eight tissue samples, with an approximate diameter of 1 to 3 $\mathrm{mm}$ each, were analyzed. Histological examinations revealed mild inflammatory mucosal lesions of the ampulla and duodenum, with regional gastric metaplasia, regional epithelial hyperplasia, and mild nuclear stratification, which did not meet the diagnostic criteria for epithelial dysplasia.

\section{DISCUSSION}

The majority of periampullary neoplasms are malignant, whereas benign neoplasms account for less than $10 \%$ of the cases. Among benign lesions, adenomas are the most common, with a potential for malignant transformation. Although biopsies are essential in the diagnosis and follow-up of 
these lesions, the histological distinction between normal and pathological features of the ampullary mucosa is often difficult to make on endoscopically obtained samples. ${ }^{1}$

Acute pancreatitis is a well-recognized complication of endoscopic retrograde cholangiopancreatography, but is extremely rare after biopsy of the ampulla of Vater without a previous attempt at cannulation. However, an asymptomatic increase in amylase concentration has been reported in up to $30 \%$ of cases. ${ }^{2}$

In the case we present, the diagnosis of acute pancreatitis was based on symptomatology in combination with the increase in serum and urine amylase levels, as well as the CT findings. To our knowledge, there are only three reported cases associating acute pancreatitis with endoscopic biopsies from the ampulla of Vater. The first case was reported in a patient with Gardner syndrome, who developed acute progressive periumbilical pain radiating laterally to the back 4 hours after endoscopy, with temperature increasing up to $39.2^{\circ} \mathrm{C}$ on the following day, high serum amylase levels, and a CT scan that showed pancreatic enlargement and diffuse stranding of the peripancreatic fat. ${ }^{3}$

Another case of severe acute pancreatitis was attributed to endoscopic biopsies of the minor papilla in a patient with pancreas divisum and liver metastases due to colon cancer. ${ }^{4}$ The third was also a case of severe pancreatitis with formation of a large pancreatic walled-off necrosis that was managed with ultrasonography-guided drainage. ${ }^{5}$

In all the aforementioned cases, abdominal pain was the presenting symptom and occurred 4 hours after sampling of the papilla. The clinical profile and course of our patient was similar to the case with Gardner syndrome, with intense symptoms but still not a severe form of pancreatitis.

Intramural duodenal hematoma due to biopsies may lead to pancreatitis associated with obstructive jaundice. ${ }^{6}$ Duodenal intramural hematoma is a very rare condition, usually related to trauma. The risk is additionally increased if anticoagulants are involved. Our patient was indeed on heparin up to 24 hours before the procedure, which in combination with the biopsy process could have led to the development of a small hematoma. However, no relevant lesion was found on CT.

Our patient was sickle cell heterozygous with a history of pulmonary embolism. Patients with sickle cell trait, especially African-Americans, are more prone to manifest deep vein thrombosis and pulmonary embolism, although this remains a matter of debate. Vaso-occlusive phenomena have been described in patients with sickle cell trait (splenic infarction or sequestration, hyphema, etc.). ${ }^{8}$ The occurrence of a thrombotic event would have led to ischemic pancreatitis, which is also a very rare condition and is usually associated with a major circulatory event such as hemorrhagic shock.

Mucosal edema due to biopsies is the most plausible mechanism for pancreatitis in our case. We tried to direct the biopsies to the area around the orifice, but due to edema and bleeding after taking the first samples, it is sometimes very difficult to ensure that one or more biopsies are not directed towards the orifice. The edema may have led to increased pancreatic duct pressure. This same mechanism is also proposed by Ishida et al. ${ }^{5}$ and Morales and Hixson, ${ }^{3}$ who reported cases of acute pancreatitis following endoscopic biopsy of the ampulla. Ishida et al. ${ }^{5}$ additionally mentioned that the rise in the pancreatic duct pressure must have been low and of short duration, as no dilation of the pancreatic duct was observed.

Despite the clear chronological association between sampling of the ampulla and acute pancreatitis in our patient, we first looked for other etiologies that may have caused pancreatic inflammation. Our patient was not an excessive drinker. We carefully reviewed the patient's drug and other medical history. No use of nonsteroidal anti-inflammatory drugs, paracetamol, proton pump inhibitors, antibiotics, or other commonly used medications were reported. We could not find any relevant references with respect to low molecular weight heparin. The patient had taken acenocoumarol uneventfully for a long time. Propofol has rarely been associated with pancreatitis if large doses are given in patients with coexistent hypertriglyceridemia. Our patient was given only $60 \mathrm{mg}$ of propofol. ${ }^{9}$ When we reviewed both the patient's older and recent blood tests, no evidence of pathological values for lipids and/or calcium was found. To our knowledge, there is no association of flumazenil with pancreatitis, whereas midazolam-induced pancreatitis reports has been scarcely reported and is not well-documented. Additionally, no family history of pancreatitis was noted.

Finally, it is worth mentioning that our patient recalled having substantial epigastric discomfort that lasted for 24 hours after a previous upper endoscopy when biopsies from the ampulla had been taken. One can assume that mild forms of pancreatitis may occur more often than is actually reported in the literature.

In conclusion, we present a case of acute pancreatitis following biopsy of the ampulla. The case is significant for certain characteristics that have not been described in similar cases. Our patient's symptomatology was severe and not in parallel with the trivial laboratory and radiological pathology, and most importantly, with the excellent outcome. The patient's medical history entails features of unknown importance for the pathogenesis of pancreatitis (sickle-cell trait, previous use of anticoagulants, and repeat sampling), but these features may have contributed to the advent of pancre- 
atitis; therefore, this case may be worth reporting for future reference. Finally, although pancreatitis after sampling of the ampulla is a rare complication, endoscopists should take it into consideration before the procedure and inform patients accordingly.

Conflicts of Interest

The authors have no financial conflicts of interest.

\section{REFERENCES}

1. Bellizzi AM, Kahaleh M, Stelow EB. The assessment of specimens procured by endoscopic ampullectomy. Am J Clin Pathol 2009;132:506-513.

2. Saurin JC, Gutknecht C, Napoleon B, et al. Surveillance of duodenal adenomas in familial adenomatous polyposis reveals high cumulative risk of advanced disease. J Clin Oncol 2004;22:493-498.

3. Morales TG, Hixson LJ. Acute pancreatitis following endoscopic biopsy of the ampulla in a patient with Gardner's syndrome. Gastrointest Endosc 1994;40:367-369.

4. Gincul R, Ciocirlan M, Dumortier J, et al. Severe acute pancreatitis following endoscopic biopsy of the minor duodenal papilla. Endoscopy 2009;41 Suppl 2:E195-E196.

5. Ishida $\mathrm{Y}$, Okabe $\mathrm{Y}$, Tokuyasu $\mathrm{H}$, et al. A case of acute pancreatitis following endoscopic biopsy of the ampulla of Vater. Kurume Med J 2013;60:67-70

6. Zinelis SA, Hershenson LM, Ennis MF, Boller M, Ismail-Beigi F. Intramural duodenal hematoma following upper gastrointestinal endoscopic biopsy. Dig Dis Sci 1989;34:289-291.

7. Folsom AR, Tang W, Roetker NS, et al. Prospective study of sickle cell trait and venous thromboembolism incidence. J Thromb Haemost 2015;13:2-9.

8. Gharaibeh A, Savage HI, Scherer RW, Goldberg MF, Lindsley K. Medical interventions for traumatic hyphema. Cochrane Database Syst Rev 2013;(12):CD005431.

9. Devlin JW, Lau AK, Tanios MA. Propofol-associated hypertriglyceridemia and pancreatitis in the intensive care unit: an analysis of frequency and risk factors. Pharmacotherapy 2005;25:1348-1352. 